\title{
Quantitative Proteomic changes after Organophosphorous Nerve Agent Exposure in the Rat Hippocampus
}

Naveen Singh ${ }^{1}$, RamaRao Golime ${ }^{1 *}$, Jyothiranjan Acharya ${ }^{2}$ and Meehir Palit

${ }^{1}$ Biochemistry-Vertox Division, Defence Research and Development Establishment, Jhansi road, Gwalior, M.P. India. ${ }^{2}$ Process Technology Development Division, DRDE, Gwalior, M.P. India.

*Corresponding author: RamaRao Golime, ramugolime@ gmail.com

This document contains the list of proteins upregulated proteins (A) and downregulated proteins (B) at $30 \mathrm{~min}$ in the rat hippocampus after soman exposure by LC-MS/MS analysis.

A). Upregulated proteins at $30 \mathrm{~min}$

\begin{tabular}{|c|c|c|}
\hline Accession & Description & $\begin{array}{l}\text { Fold } \\
\text { change }\end{array}$ \\
\hline F1LTL7 & Protein RGD1559662 GN=RGD1559662 & 2.907 \\
\hline D4A9P8 & Protein Chst5 GN=Chst5 & 2.858 \\
\hline G3V7Z6 & ATP-binding cassette sub-family $\mathrm{D}$ member $2 \mathrm{GN}=\mathrm{Abcd} 2$ & 2.733 \\
\hline Q8K3H3 & NPT1 GN=Slc17a1 & 2.647 \\
\hline F1M376 & Olfactory receptor (Fragment) GN=Olfr1102 & 2.51 \\
\hline D3ZS95 & Protein Dpy19|4 (Fragment) GN=Dpy19|4 & 2.469 \\
\hline E9PSK5 & Protein Sik2 GN=Sik2 & 2.417 \\
\hline Q66HD3 & Nuclear autoantigenic sperm protein $\mathrm{GN}=\mathrm{Nasp}$ & 2.258 \\
\hline P51869 & Cytochrome P450 4F4 GN=Cyp4f4 & 2.198 \\
\hline F1M6D4 & Protein Flt3 (Fragment) GN=Flt3 & 2.114 \\
\hline D3ZDP2 & Protein Ict1 GN=Ict1 & 2.106 \\
\hline Q5FWT8 & REST corepressor $2 \mathrm{GN}=\mathrm{Rcor} 2$ & 2 \\
\hline F8WLE0 & Kinesin-like protein KIF28P GN=Kif28p & 1.992 \\
\hline Q9R1U9 & Beta-1,3-N-acetylglucosaminyltransferase radical fringe $\mathrm{GN}=\mathrm{Rfng}$ & 1.966 \\
\hline G8CYZ7 & Engulfment and cell motility 1 splice 1 variant GN=Elmo1 & 1.956 \\
\hline D3ZYQ8 & Uridine-cytidine kinase $\mathrm{GN}=\mathrm{Uckl} 1$ & 1.952 \\
\hline D3ZMN5 & Protein $\mathrm{Sn} \times 29 \mathrm{GN}=\mathrm{Sn} \times 29$ & 1.912 \\
\hline BOBNJ7 & Baz2b protein (Fragment) GN=Baz2b & 1.902 \\
\hline Q6TXG4 & LRRGT00035 GN=Dhx8 & 1.902 \\
\hline D4AEN1 & Protein Rtp1 GN=Rtp1 & 1.877 \\
\hline D4ABM3 & Dishevelled associated activator of morphogenesis 1 (Predicted) GN=Daam1 & 1.872 \\
\hline F1LQT1 & Adenylate cyclase $7 \mathrm{GN}=\mathrm{Adcy} 7$ & 1.87 \\
\hline M0RA38 & Protein NIrp2 GN=LOC684545 & 1.869 \\
\hline Q9Z286 & Adenylate cyclase type $10 \mathrm{GN}=\mathrm{Adcy} 10$ & 1.868 \\
\hline D3ZCK4 & Protein Def6 GN=Def6 & 1.866 \\
\hline Q63418 & Protocadherin $-3 \mathrm{GN}=$ Pcdh3 & 1.819 \\
\hline D3ZFA2 & Protein Nek10 GN=Nek10 & 1.81 \\
\hline
\end{tabular}




\begin{tabular}{|c|c|c|}
\hline P56718 & Orexin receptor type $1 \mathrm{GN}=\mathrm{Hcrtr} 1$ & 1.789 \\
\hline D4A8Z5 & Protein Mett|21a GN=Mett|21a PE=4 SV=1 & 1.789 \\
\hline D4A704 & Protein Zfp641 GN=Zfp641 & 1.778 \\
\hline D4A3P4 & Nucleolar protein 4 (Predicted), isoform CRA_b GN=Nol4 & 1.768 \\
\hline D3ZTZO & Protein Fam184b GN=Fam184b & 1.761 \\
\hline G3V8U6 & DNA polymerase alpha subunit $B$ GN=Pola2 & 1.751 \\
\hline Q6AYK9 & Chromodomain Y-like protein $\mathrm{GN}=\mathrm{Cdyl}$ & 1.736 \\
\hline Q7TP57 & Signal transducer and activator of transcription GN=Stat4 & 1.735 \\
\hline D3ZID8 & Protein Ubxn2a GN=Ubxn2a & 1.732 \\
\hline D3ZGJ9 & Protein Depdc1 GN=Depdc1 & 1.712 \\
\hline Q66H47 & 39S ribosomal protein L24, mitochondrial GN=Mrpl24 & 1.7 \\
\hline Q9WUI9 & Nuclear receptor coactivator $2 \mathrm{GN}=\mathrm{Ncoa} 2$ & 1.697 \\
\hline Q3B7U0 & Ripk2 protein (Fragment) GN=Ripk2 & 1.694 \\
\hline D3ZKG3 & Protein Sec14l1 GN=Sec14l1 & 1.693 \\
\hline D3ZMU0 & Protein Slc35g1 GN=Slc35g1 & 1.689 \\
\hline Q5U2R1 & Decaprenyl-diphosphate synthase subunit $2 \mathrm{GN}=\mathrm{Pdss} 2$ & 1.687 \\
\hline G3V7A5 & Low density lipoprotein receptor, isoform CRA_a GN=Ldlr & 1.68 \\
\hline Q3T119 & RNA polymerase II-associated protein $1 \mathrm{GN}=\mathrm{Rpap} 1 \mathrm{SV}=1$ & 1.667 \\
\hline D4A4L5 & Protein Isca2 GN=Isca2 & 1.664 \\
\hline Q7TP90 & Arrestin domain-containing protein $4 \mathrm{GN}=\mathrm{Arrdc} 4$ & 1.657 \\
\hline D3ZS69 & Protein $\mathrm{C} 2 \mathrm{~cd} 3 \mathrm{GN}=\mathrm{C} 2 \mathrm{~cd} 3$ & 1.655 \\
\hline D4AB49 & Protein Prss33 GN=Prss33 & 1.654 \\
\hline D3ZXL4 & Protein Rsph3 GN=Rsph3 & 1.647 \\
\hline D3ZXE3 & Protein Tbc1d9 GN=Tbc1d9 & 1.644 \\
\hline D3ZDN7 & Protein Fgd6 GN=Fgd6 & 1.642 \\
\hline Q62771 & Signal transducer and activator of transcription $5 \mathrm{~A} \mathrm{GN=Stat5a}$ & 1.637 \\
\hline B1WC58 & DNA endonuclease RBBP8 GN=Rbbp8 & 1.609 \\
\hline P13109 & Fibroblast growth factor $2 \mathrm{GN}=\mathrm{Fgf2}$ & 1.601 \\
\hline D3ZYR1 & FCH domain only protein $2 \mathrm{GN}=\mathrm{Fcho} 2$ & 1.6 \\
\hline $\mathrm{B} 1 \mathrm{H} 241$ & Resistance to inhibitors of cholinesterase 8 homolog A (C. elegans) GN=Ric8a & 1.593 \\
\hline D3ZV19 & Protein Tmem64 GN=Tmem64 & 1.593 \\
\hline F1M9X1 & Sodium channel protein type 11 subunit alpha $\mathrm{GN}=\mathrm{Scn} 11 \mathrm{a}$ & 1.589 \\
\hline Q2VW85 & CD6deltaD3 (Fragment) & 1.582 \\
\hline B5DEZ8 & Plexin domain containing $2 \mathrm{GN}=\mathrm{Plxdc2}$ & 1.573 \\
\hline BOBNB1 & Commd1 protein $\mathrm{GN}=$ Commd1 & 1.566 \\
\hline D3ZQG0 & Protein Rnmtl1 GN=Rnmtl1 & 1.553 \\
\hline F1M3R4 & Unconventional myosin-Vb GN=Myo5b & 1.547 \\
\hline D4A7T9 & Protein Mysm1 GN=Mysm1 & 1.545 \\
\hline Q3T112 & Complement C1q tumor necrosis factor-related protein $6 \mathrm{GN}=\mathrm{C} 1 \mathrm{qtnf} 6$ & 1.541 \\
\hline G3V9T1 & Protein Crispld2 GN=Crispld2 & 1.529 \\
\hline P62718 & $60 \mathrm{~S}$ ribosomal protein $\mathrm{L} 18 \mathrm{a} \mathrm{GN}=\mathrm{Rp} 118 \mathrm{a}$ & 1.528 \\
\hline
\end{tabular}




\begin{tabular}{|l|l|l|}
\hline D4A3X0 & Protein Seh1I GN=Seh1I & 1.527 \\
\hline D4AAM1 & Protein LOC690349 GN=LOC690349 & 1.526 \\
\hline Q5FWS6 & Kazrin GN=Kazn & 1.523 \\
\hline D3ZBH5 & Protein Lmtk2 GN=Lmtk2 & 1.511 \\
\hline
\end{tabular}

B). Downregulated proteins at $30 \mathrm{~min}$

\begin{tabular}{|c|c|c|}
\hline Accession & Description & Fold change \\
\hline F1M4Q3 & Protein Hmcn1 GN=Hmcn1 & 0.666 \\
\hline Q4V819 & Protein Ugp2 GN=Ugp2 PE=2 SV=1 & 0.665 \\
\hline P13852 & Major prion protein $\mathrm{GN}=\operatorname{Prnp}$ & 0.665 \\
\hline F1M285 & Protein Bbs9 (Fragment) GN=Bbs9 & 0.663 \\
\hline P97706 & Sodium channel GN=Scn7a & 0.662 \\
\hline B6ZGR5 & Melanocortin-1 receptor $\mathrm{GN}=\mathrm{Mc1r}$ & 0.661 \\
\hline Q5M9G3 & Caprin-1 GN=Caprin1 & 0.659 \\
\hline Q9Z269 & Vesicle-associated membrane protein-associated protein B GN=Vapb & 0.657 \\
\hline G3V8R0 & Protein RGD1311703 GN=RGD1311703 & 0.656 \\
\hline Q66H99 & Nucleolar protein $10 \mathrm{GN}=\mathrm{Nol} 10$ & 0.652 \\
\hline F1LQ45 & Epsin-2 GN=Epn2 & 0.65 \\
\hline D3ZL07 & Hypothetical LOC292874 (Predicted), isoform CRA_b GN=RGD1309036 & 0.65 \\
\hline P97612 & Fatty-acid amide hydrolase $1 \mathrm{GN}=$ Faah & 0.648 \\
\hline F1M0J5 & Protein Hpse2 (Fragment) GN=Hpse2 & 0.648 \\
\hline Q00960 & Glutamate receptor ionotropic, NMDA 2B GN=Grin2b & 0.647 \\
\hline Q5M951 & UPF0688 protein C1orf174 homolog & 0.644 \\
\hline F1M897 & Protein Col22a1 GN=Col22a1 & 0.642 \\
\hline B5DEY4 & Mitochondrial ribosomal protein $\mathrm{L} 30 \mathrm{GN}=\mathrm{Mrpl} 30$ & 0.641 \\
\hline D4A1K4 & Hypothetical LOC304650 (Predicted), isoform CRA_b GN=RGD1310262 & 0.641 \\
\hline Q5J3M9 & Vomeronasal type-1 receptor $97 \mathrm{GN}=$ Vom1r97 & 0.641 \\
\hline D4ABU4 & Protein Poc1a GN=Poc1a & 0.637 \\
\hline P63012 & Ras-related protein Rab-3A GN=Rab3a & 0.636 \\
\hline D3ZVU1 & SprT-like domain-containing protein Spartan GN=Sprtn & 0.632 \\
\hline M0R8A5 & Protein Ca10 GN=Ca10 & 0.631 \\
\hline M0R9Z5 & Protein Irf2bp2 GN=Irf2bp2 & 0.63 \\
\hline D3ZJF7 & Protein Terf2 GN=Terf2 & 0.629 \\
\hline Q8K3K4 & Serpin B10 GN=Serpinb10 & 0.628 \\
\hline D4A355 & Protein Mastl GN=Mastl & 0.628 \\
\hline P18665 & $39 \mathrm{~S}$ ribosomal protein $\mathrm{L} 3$, mitochondrial $\mathrm{GN}=\mathrm{Mrpl} 3$ & 0.627 \\
\hline D3ZAZ5 & Breast cancer anti-estrogen resistance 3 (Predicted) GN=Bcar3 & 0.625 \\
\hline D3ZC19 & DNA (cytosine-5)-methyltransferase $1 \mathrm{GN}=\mathrm{Dnmt1}$ & 0.624 \\
\hline Q68FX9 & NAD-dependent protein deacylase sirtuin-5, mitochondrial GN=Sirt5 & 0.623 \\
\hline B6VQA5 & Spermatogenesis-associated protein $31 \mathrm{GN}=$ Spata31 & 0.621 \\
\hline
\end{tabular}




\begin{tabular}{|c|c|c|}
\hline F1M495 & Mannose receptor, C type 2 (Predicted) GN=Pla2r1 & 0.62 \\
\hline P70583 & Deoxyuridine 5'-triphosphate nucleotidohydrolase GN=Dut & 0.619 \\
\hline Q5XIM5 & Protein CDV3 homolog $\mathrm{GN}=\mathrm{Cdv} 3$ & 0.617 \\
\hline Q8QZZ9 & Cyclin-dependent kinase inhibitor $2 \mathrm{~A}$, isoform $2 \mathrm{GN}=\mathrm{Cdkn} 2 \mathrm{a}$ & 0.603 \\
\hline F7ENY6 & Protein Als2cr11 GN=Als2cr11 & 0.602 \\
\hline Q5M853 & KxDL motif-containing protein $1 \mathrm{GN}=\mathrm{Kxd} 1$ & 0.601 \\
\hline P60042 & Somatostatin $\mathrm{GN}=\mathrm{Sst}$ & 0.599 \\
\hline D4A5K9 & Glycogen [starch] synthase, liver GN=Gys2 & 0.598 \\
\hline Q7TP53 & $\mathrm{Ab2}-183 \mathrm{GN}=\| 12 \mathrm{rg}$ & 0.594 \\
\hline Q05175 & Brain acid soluble protein $1 \mathrm{GN}=$ Basp1 & 0.588 \\
\hline P13941 & Collagen alpha-1(III) chain GN=Col3a1 & 0.588 \\
\hline D3Z8M1 & Olfactory receptor GN=Olr1452 & 0.586 \\
\hline Q6TXF5 & Leucine-rich repeat-containing protein $66 \mathrm{GN}=\mathrm{Lrrc} 66$ & 0.584 \\
\hline D3ZLN6 & Protein Trim43a GN=Trim43a & 0.577 \\
\hline M0R6H6 & Protein 9330101J02Rik GN=RGD1559985 & 0.577 \\
\hline D4ABL9 & Protein Ccdc151 GN=Ccdc151 & 0.575 \\
\hline Q5U313 & Ankyrin repeat domain $13 a \mathrm{GN}=\mathrm{Ankrd} 13 \mathrm{a}$ & 0.562 \\
\hline B4F7E5 & ATPase, $\mathrm{Ca}++$ transporting, cardiac muscle, fast twitch $1 \mathrm{GN}=$ Atp2a1 & 0.561 \\
\hline Q5BJN2 & Death-associated kinase $2 \mathrm{GN}=\mathrm{Dapk} 2$ & 0.561 \\
\hline Q7TMA5 & Apolipoprotein B-100 GN=Apob & 0.546 \\
\hline F1M1A6 & Protein LOC681355 GN=LOC681355 & 0.545 \\
\hline P31421 & Metabotropic glutamate receptor $2 \mathrm{GN}=\mathrm{Grm} 2$ & 0.545 \\
\hline Q9R011 & Serine/threonine-protein kinase PLK3 GN=PIk3 & 0.538 \\
\hline D3ZTX7 & Ubiquitin carboxyl-terminal hydrolase $\mathrm{GN}=\mathrm{Usp} 22$ & 0.532 \\
\hline D4ABA6 & Protein Actrt3 OS=Rattus norvegicus GN=Actrt3 & 0.526 \\
\hline Q19P45 & Cytochrome P450Scc (Fragment) GN=Cyp11a1 & 0.526 \\
\hline D4A9J6 & Protein Ccdc88b GN=Ccdc88b & 0.522 \\
\hline P47967 & Galectin-5 GN=Lgals5 & 0.514 \\
\hline P02454 & Collagen alpha-1(I) chain GN=Col1a1 & 0.51 \\
\hline Q32Q54 & Protein Uqcc GN=Uqcc1 PE=2 SV=1 & 0.448 \\
\hline Q8SEZ6 & Cytochrome c oxidase subunit $1 \mathrm{GN}=\mathrm{COI}$ & 0.403 \\
\hline Q66H58 & von Willebrand factor $A$ domain-containing protein $9 \mathrm{GN}=\mathrm{Vwa9}$ & 0.39 \\
\hline D4A558 & Protein LOC100912275 GN=Mixl1 & 0.385 \\
\hline F1LPE2 & Protein sel-1 homolog 2 (Fragment) GN=Sel1/2 & 0.355 \\
\hline
\end{tabular}


This table S2 contains the list of proteins upregulated proteins (A) and downregulated proteins (B) at 1 day in the rat hippocampus after soman exposure by LC-MS/MS analysis.

A). Upregulated proteins at 1 day

\begin{tabular}{|c|c|c|}
\hline Accession & Description & $\begin{array}{l}\text { Fold } \\
\text { change }\end{array}$ \\
\hline D3ZS95 & Protein Dpy19|4 (Fragment) GN=Dpy19|4 & 3.04 \\
\hline D3ZDP2 & Protein Ict1 $\mathrm{GN}=$ Ict1 & 2.678 \\
\hline B4F7C4 & Tudor domain-containing protein $5 \mathrm{GN}=\mathrm{Tdrd} 5$ & 2.527 \\
\hline D4A9P8 & Protein Chst5 $\mathrm{GN}=$ Chst5 & 2.409 \\
\hline D4A558 & Protein LOC100912275 GN=Mixl1 & 2.186 \\
\hline D4AAY3 & Protein Rasal2 GN=Rasal2 & 2.182 \\
\hline P42930 & Heat shock protein beta-1 $\mathrm{GN}=\mathrm{Hspb} 1$ & 2.1 \\
\hline Q66H47 & 39S ribosomal protein L24, mitochondrial GN=Mrpl24 & 2.013 \\
\hline Q5U2R1 & Decaprenyl-diphosphate synthase subunit $2 \mathrm{GN}=\mathrm{Pdss} 2$ & 2.011 \\
\hline D3ZXE3 & Protein Tbc1d9 GN=Tbc1d9 & 1.937 \\
\hline Q7M6Z5 & Kinesin-like protein KIF27 GN=Kif27 & 1.84 \\
\hline Q5RKH7 & Solute carrier family 35 member F6 $\mathrm{GN}=\mathrm{Slc} 35 \mathrm{f} 6$ & 1.84 \\
\hline B2GV55 & Protein Ube2q1 GN=Ube2q1 & 1.822 \\
\hline P26769 & Adenylatecyclase type 2 GN=Adcy 2 & 1.784 \\
\hline P81155 & Voltage-dependent anion-selective channel protein 2, GN=Vdac2 & 1.781 \\
\hline D3ZKG3 & Protein Sec14l1 GN=Sec14l1 & 1.78 \\
\hline BOBNJ7 & Baz2b protein (Fragment) GN=Baz2b & 1.778 \\
\hline F8WLE0 & Kinesin-like protein KIF28P GN=Kif28p & 1.765 \\
\hline BOBNM2 & $\mathrm{Mbd} 2$ protein $\mathrm{GN}=\mathrm{Mbd} 2$ & 1.726 \\
\hline Q62736 & Non-muscle caldesmon GN=Cald1 & 1.712 \\
\hline Q9QZG8 & Adenylyl cyclase type VII (Fragment) GN=Adcy7 & 1.698 \\
\hline Q32Q54 & Protein Uqcc GN=Uqcc1 & 1.683 \\
\hline Q7TP90 & Arrestin domain-containing protein $4 \mathrm{GN}=\mathrm{Arrdc} 4$ & 1.674 \\
\hline F1LV37 & Protein Tnrc6b GN=Tnrc6b & 1.669 \\
\hline D3ZJF7 & Protein Terf2 GN=Terf2 & 1.667 \\
\hline A1A5S4 & Protein Sephs2 GN=Sephs2 & 1.665 \\
\hline F1M9X1 & Sodium channel protein type 11 subunit alpha GN=Scn11a & 1.658 \\
\hline F1LQT1 & Adenylatecyclase $7 \mathrm{GN}=\mathrm{Adcy} 7$ & 1.645 \\
\hline D3ZHV2 & Microtubule-actin cross-linking factor $1 \mathrm{GN}=$ Macf1 & 1.64 \\
\hline Q6PPF3 & Harmonin a1 GN=Ush1c & 1.626 \\
\hline B1WC58 & DNA endonuclease RBBP8 GN=Rbbp8 & 1.625 \\
\hline D3ZS69 & Protein $\mathrm{C} 2 \mathrm{~cd} 3 \mathrm{GN}=\mathrm{C} 2 \mathrm{~cd} 3$ & 1.624 \\
\hline D4A3X0 & Protein Seh1l GN=Seh1l & 1.613 \\
\hline Q6XQG8 & Carbohydrate sulfotransferase $7 \mathrm{GN}=$ Chst7 & 1.611 \\
\hline D3ZQ18 & Protein Nccrp1 GN=Nccrp1 & 1.603 \\
\hline
\end{tabular}




\begin{tabular}{|c|c|c|}
\hline P22057 & Prostaglandin-H2 D-isomerase GN=Ptgds & 1.602 \\
\hline D7NIWO & Nedd4l & 1.6 \\
\hline D3ZBH5 & Protein Lmtk2 GN=Lmtk2 & 1.597 \\
\hline B5DEZ8 & Plexin domain containing $2 \mathrm{GN}=\mathrm{Plxdc2}$ & 1.587 \\
\hline Q62839 & Golgin subfamily A member 2 GN=Golga2 & 1.584 \\
\hline B1WBR5 & Mobp protein $\mathrm{GN}=$ Mobp & 1.581 \\
\hline EOA3N4 & Serpina3n-like protein & 1.578 \\
\hline Q9Z286 & Adenylatecyclase type $10 \mathrm{GN}=\mathrm{Adcy} 10$ & 1.577 \\
\hline D3ZYX5 & Protein Myo6 GN=Myo6 & 1.574 \\
\hline 088764 & Death-associated protein kinase 3 GN=Dapk3 & 1.567 \\
\hline D4A7T8 & Protein Fam81a GN=Fam81a & 1.565 \\
\hline D4A039 & Protein RGD1311344 GN=Dzank1 & 1.564 \\
\hline P11517 & Hemoglobin subunit beta-2 & 1.555 \\
\hline D3ZFJ6 & Lactamase, beta (Predicted) GN=Lactb & 1.551 \\
\hline Q66HD3 & Nuclear autoantigenic sperm protein $\mathrm{GN}=\mathrm{Nasp}$ & 1.55 \\
\hline P70500 & CDP-diacylglycerol--inositol 3-phosphatidyltransferase GN=Cdipt & 1.546 \\
\hline G3V926 & Conserved helix-loop-helix ubiquitous kinase (Predicted) GN=Chuk & 1.542 \\
\hline Q6IV68 & Fanconi anemia group D2 protein homolog GN=Fancd2 & 1.538 \\
\hline P08482 & Muscarinic acetylcholine receptor M1 GN=Chrm1 & 1.536 \\
\hline F1M790 & Prostaglandin F2 receptor negative regulator (Fragment) GN=Ptgfrn & 1.532 \\
\hline D4A6P1 & Protein Sez6l2 OS=Rattusnorvegicus GN=Sez6l2 & 1.529 \\
\hline D3ZIF5 & $\begin{array}{l}\text { Adaptor-related protein complex } 3 \text {, beta } 1 \text { subunit (Predicted), isoform CRA_b } \\
\text { GN=Ap3b1 }\end{array}$ & 1.526 \\
\hline F1MA52 & Nuclear RNA export factor $1 \mathrm{GN}=\mathrm{Nxf1}$ & 1.524 \\
\hline D3ZKD4 & Protein Cdt1 $\mathrm{GN}=\mathrm{Cdt} 1$ & 1.523 \\
\hline F1LTC6 & Protein $\mathrm{Gm} 8787$ (Fragment) GN=Gm8787 & 1.522 \\
\hline M0RA86 & Protein Ccdc9 GN=Ccdc9 & 1.515 \\
\hline P20156 & Neurosecretory protein VGF GN=Vgf & 1.513 \\
\hline 008836 & Immunoglobulin-binding protein $1 \mathrm{GN}=$ Igbp 1 & 1.51 \\
\hline
\end{tabular}


B). Downregulated proteins at 1 day

\begin{tabular}{|c|c|c|}
\hline Accession & Description & $\begin{array}{l}\text { Fold } \\
\text { change }\end{array}$ \\
\hline M0RBW5 & Protein Ccdc40 GN=Ccdc40 & 0.665 \\
\hline Q6P9T8 & Tubulin beta-4B chain $\mathrm{GN}=\mathrm{Tubb} 4 \mathrm{~b}$ & 0.664 \\
\hline I7FKL4 & Myelin basic protein transcript variant $1 \mathrm{GN}=\mathrm{Mbp}$ & 0.663 \\
\hline Q5U313 & Ankyrin repeat domain 13a GN=Ankrd13a & 0.662 \\
\hline F1LWD7 & Protein Klhl32 GN=Klhl32 & 0.662 \\
\hline Q5XFW6 & WD repeat-containing protein $6 \mathrm{GN}=\mathrm{Wdr} 6$ & 0.654 \\
\hline Q7TMA5 & Apolipoprotein B-100 GN=Apob & 0.653 \\
\hline D4A9H8 & Protein Nim1 GN=Nim1 & 0.648 \\
\hline P60203 & Myelin proteolipid protein GN=Plp1 & 0.639 \\
\hline M0R6H6 & Protein 9330101J02Rik GN=RGD1559985 & 0.639 \\
\hline B5DEG7 & Protein Champ1 GN=Champ1 & 0.637 \\
\hline Q9R011 & Serine/threonine-protein kinase PLK3 GN=PIk3 & 0.634 \\
\hline P02454 & Collagen alpha-1(I) chain GN=Col1a1 & 0.625 \\
\hline P29293 & Acrosin $\mathrm{GN}=\mathrm{Acr}$ & 0.625 \\
\hline F1MAG7 & Protein Ptpru GN=Ptpru & 0.623 \\
\hline D4ABL9 & Protein Ccdc151 GN=Ccdc151 & 0.62 \\
\hline D3ZJL1 & Malignant fibrous histiocytoma amplified sequence 1 (Predicted), isoform & 0.618 \\
\hline P31421 & Metabotropic glutamate receptor $2 \mathrm{GN}=\mathrm{Grm} 2$ & 0.61 \\
\hline 070467 & Protein arginine $\mathrm{N}$-methyltransferase $3 \mathrm{GN}=\mathrm{Prmt} 3$ & 0.608 \\
\hline D3ZD19 & Extracellular link domain-containing 1 (Predicted) GN=Lyve1 & 0.608 \\
\hline D3ZUE4 & Protein Socs5 GN=Socs5 & 0.594 \\
\hline B6VQA5 & Spermatogenesis-associated protein $31 \mathrm{GN}=$ Spata31 & 0.593 \\
\hline Q3KR54 & Adenosine deaminase domain-containing protein $1 \mathrm{GN}=\mathrm{Adad} 1$ & 0.593 \\
\hline Q6AY08 & $\begin{array}{l}\text { Amyotrophic lateral sclerosis } 2 \text { chromosomal region candidate gene } 12 \\
\text { protein homolog } \mathrm{GN}=\mathrm{Als} 2 \mathrm{cr} 12\end{array}$ & 0.593 \\
\hline A1A5R2 & G protein-coupled receptor, family C, group 5 , member A GN=Gprc5a & 0.592 \\
\hline M0R576 & Protein $\mathrm{Cd} 247 \mathrm{GN}=\mathrm{Cd} 247$ & 0.586 \\
\hline D3ZTX7 & Ubiquitin carboxyl-terminal hydrolase GN=Usp22 & 0.577 \\
\hline D4A355 & Protein Mastl GN=Mastl & 0.577 \\
\hline P47967 & Galectin-5 GN=Lgals5 & 0.573 \\
\hline Q3T1L0 & Aldehyde dehydrogenase family 16 member A1 GN=Aldh16a1 & 0.568 \\
\hline E9PTYO & Protein Lrrcc1 GN=Lrrcc1 & 0.565 \\
\hline D4A9J6 & Protein Ccdc88b GN=Ccdc88b & 0.528 \\
\hline D4A5K9 & Glycogen [starch] synthase, liver GN=Gys2 & 0.515 \\
\hline Q63207 & Coagulation factor $\mathrm{X} \mathrm{GN}=\mathrm{F} 10$ & 0.46 \\
\hline D3ZED9 & Protein Lrrc10b GN=Lrrc10b & 0.42 \\
\hline Q499S5 & Stromelysin-3 GN=Mmp11 & 0.414 \\
\hline F1LPE2 & Protein sel-1 homolog 2 (Fragment) GN=Sel1/2 & 0.329 \\
\hline
\end{tabular}


This Table S3 contains the list of proteins upregulated proteins (A) and downregulated proteins (B) at 7 day in the rat hippocampus after soman exposure by LC-MS/MS analysis.

A). Upregulated proteins at 7 day

\begin{tabular}{|c|c|c|}
\hline Accession & Description & $\begin{array}{l}\text { Fold } \\
\text { change }\end{array}$ \\
\hline Q8SEZ6 & Cytochrome $\mathrm{c}$ oxidase subunit $1 \mathrm{GN}=\mathrm{COI}$ & 3.589 \\
\hline D4AAA5 & Fanconi anemia-associated protein of $20 \mathrm{kDa}$ GN=Faap20 & 3.346 \\
\hline Q6PPF3 & Harmonin a1 GN=Ush1c & 2.887 \\
\hline F1M7D8 & Protein Gramd4 (Fragment) GN=Gramd4 & 2.588 \\
\hline D4A3E0 & Protein Mmrn1 GN=Mmrn1 & 2.56 \\
\hline P26769 & Adenylatecyclase type $2 \mathrm{GN}=\mathrm{Adcy} 2$ & 2.316 \\
\hline F1M859 & Protein Cphx (Fragment) GN=Cphx & 2.306 \\
\hline M0R7D8 & Protein Suz12 GN=Suz12 & 2.221 \\
\hline D4A7T8 & Protein Fam81a GN=Fam81a & 1.904 \\
\hline F1MAL0 & Protein Zfp266 GN=Zfp266 & 1.881 \\
\hline Q5S6T3 & Isoprenoid synthase domain-containing protein $\mathrm{GN}=\mathrm{Ispd}$ & 1.873 \\
\hline P02454 & Collagen alpha-1(I) chain GN=Col1a1 & 1.866 \\
\hline D4ABB3 & Protein Adamts17 GN=Adamts17 & 1.819 \\
\hline P15800 & Laminin subunit beta- $2 \mathrm{GN}=\mathrm{Lamb} 2$ & 1.818 \\
\hline Q62835 & RabGTPase-binding effector protein 2 GN=Rabep2 & 1.777 \\
\hline D3ZMB0 & $\begin{array}{l}\text { A disintegrin-like and metalloprotease (Reprolysin type) with thrombospondin type } 1 \\
\text { motif, } 9 \text { (Predicted) GN=Adamts9 }\end{array}$ & 1.77 \\
\hline D4A0L2 & Protein Dmrta2 GN=Dmrta2 & 1.749 \\
\hline G3V9Q6 & $\begin{array}{l}\text { Similar to putative TRAF and TNF receptor associated protein, isoform CRA_c } \\
\text { GN=Tdp2 }\end{array}$ & 1.737 \\
\hline Q66HB3 & Intraflagellar transport protein 80 homolog $\mathrm{GN}=\mathrm{Ift} 80$ & 1.733 \\
\hline F1LS02 & Nuclear pore complex protein Nup155 GN=Nup155 & 1.71 \\
\hline F1M886 & Protein $\operatorname{Oog} 3 \mathrm{GN}=\mathrm{Oog} 3$ & 1.706 \\
\hline D4A2F6 & Protein Nhlrc3 GN=Nhlrc3 & 1.687 \\
\hline Q5J3F0 & Protein Vom1r109 GN=Vom1r109 & 1.682 \\
\hline Q9QYH7 & Appetite-regulating hormone $\mathrm{GN}=\mathrm{Ghrl}$ & 1.667 \\
\hline Q63460 & Proline-rich protein (Fragment) GN=PRP & 1.653 \\
\hline D4ADZ3 & Protein LOC100362344 GN=LOC100362344 & 1.64 \\
\hline D3ZD72 & Protein Ncapg (Fragment) GN=Ncapg & 1.636 \\
\hline D4A4N9 & Protein LOC685849 GN=LOC685849 & 1.635 \\
\hline H7C5X3 & Protein RGD1566078 GN=RGD1566078 & 1.617 \\
\hline F1M748 & Protein Col24a1 GN=Col24a1 & 1.602 \\
\hline F1MAG7 & Protein PtpruGN=Ptpru & 1.601 \\
\hline D3ZDR4 & Olfactory receptor $\mathrm{GN}=\mathrm{O} \operatorname{lr} 1682$ & 1.6 \\
\hline F1M9Y1 & Olfactory receptor $\mathrm{GN}=\mathrm{Olr} 1505$ & 1.599 \\
\hline D3ZSD8 & Protein Tmem143 GN=Tmem143 & 1.598 \\
\hline
\end{tabular}




\begin{tabular}{|c|c|c|}
\hline M0RCR3 & Protein Vrtn GN=Vrtn & 1.596 \\
\hline D3ZFP6 & Protein Ticrr GN=Ticrr & 1.595 \\
\hline Q76K24 & Ankyrin repeat domain-containing protein $46 \mathrm{GN}=\mathrm{Ankrd} 46$ & 1.593 \\
\hline Q8HWI2 & MHC class Ib antigen (Fragment) GN=RT1-T18 & 1.593 \\
\hline Q3MHC2 & Integrator complex subunit $11 \mathrm{GN}=\mathrm{Cpsf31}$ & 1.585 \\
\hline Q5XIE0 & Acidic leucine-rich nuclear phosphoprotein 32 family member E GN=Anp32e & 1.584 \\
\hline D4A9I4 & Pentatricopeptide repeat domain 2 (Predicted) GN=Ptcd2 & 1.567 \\
\hline F1MAB8 & Protein Kif11 GN=Kif11 & 1.56 \\
\hline B5DEN3 & Abhydrolase domain containing $8 \mathrm{GN}=\mathrm{Abhd} 8$ & 1.559 \\
\hline B4F7C4 & Tudor domain-containing protein $5 \mathrm{GN}=\mathrm{Tdrd} 5 \mathrm{PE}=2 \mathrm{SV}=1$ & 1.559 \\
\hline F7EK99 & Protein Vom1r71 GN=Vom1r71 & 1.557 \\
\hline Q63377 & Sodium/potassium-transporting ATPase subunit beta-3 GN=Atp1b3 & 1.546 \\
\hline P48303 & Sphingosine 1-phosphate receptor $1 \mathrm{GN}=\mathrm{S} 1 \mathrm{pr} 1$ & 1.545 \\
\hline P07895 & Superoxide dismutase $[\mathrm{Mn}]$, mitochondrial $\mathrm{GN}=\mathrm{Sod} 2$ & 1.542 \\
\hline D3ZEK8 & Uncharacterized protein & 1.539 \\
\hline B2GV96 & Coiled-coil domain containing $115 \mathrm{GN}=\mathrm{Ccdc} 115$ & 1.534 \\
\hline Q5U2T2 & Dehydrodolichyldiphosphate synthase GN=Dhdds & 1.534 \\
\hline D3ZLK2 & Protein Rasl12 GN=Rasl12 & 1.529 \\
\hline F1LMI7 & E3 ubiquitin-protein ligase RNF123 GN=Rnf123 & 1.527 \\
\hline Q5BK06 & Cyclin-D1-binding protein $1 \mathrm{GN}=\mathrm{Ccndbp} 1$ & 1.526 \\
\hline Q6P9V1 & $\mathrm{CD} 81$ antigen $\mathrm{GN}=\mathrm{Cd} 81$ & 1.52 \\
\hline Q641X9 & 39S ribosomal protein $\mathrm{L} 9$, mitochondrial $\mathrm{GN}=\mathrm{Mrp19}$ & 1.518 \\
\hline Q3ZAV4 & GIMAP9 GN=Gimap9 & 1.518 \\
\hline Q4V8C6 & Suppressor of fused homolog GN=Sufu & 1.514 \\
\hline P09811 & Glycogen phosphorylase, liver form GN=Pygl & 1.513 \\
\hline O70453 & Putative hemeoxygenase $3 \mathrm{GN}=\mathrm{Hmox} 3$ & 1.513 \\
\hline Q9JLZ1 & Glutaredoxin-3 GN=Glrx3 & 1.508 \\
\hline F1LR33 & Lipid phosphate phosphatase-related protein type $2 \mathrm{GN}=\mathrm{Lppr} 2$ & 1.508 \\
\hline D3ZBP4 & Protein-methionine sulfoxide oxidase MICAL1 GN=Mical1 & 1.501 \\
\hline
\end{tabular}

B). Downregulated proteins at 7 day

\begin{tabular}{|l|l|l|}
\hline Accession & Description & $\begin{array}{l}\text { Fold } \\
\text { change }\end{array}$ \\
\hline F1M495 & Mannose receptor, C type 2 (Predicted) GN=Pla2r1 & 0.663 \\
\hline F1LNH0 & Protein Sema3g GN=Sema3g & 0.662 \\
\hline D3ZJF7 & Protein Terf2 GN=Terf2 & 0.661 \\
\hline P34064 & Proteasome subunit alpha type-5 GN=Psma5 & 0.658 \\
\hline P59241 & Aurora kinase A GN=Aurka & 0.658 \\
\hline P51869 & Cytochrome P450 4F4 GN=Cyp4f4 & 0.653 \\
\hline D3Z9E8 & Protein Fndc8 GN=Fndc8 & 0.652 \\
\hline D3ZPV3 & Protein RGD1566380 GN=RGD1566380 & 0.651 \\
\hline
\end{tabular}




\begin{tabular}{|c|c|c|}
\hline P97612 & Fatty-acid amide hydrolase $1 \mathrm{GN}=\mathrm{Faah}$ & 0.65 \\
\hline Q9QZQ4 & Urotensin-2 GN=Uts2 & 0.649 \\
\hline D4A8Z5 & Protein Mettl21a GN=Mettl21a & 0.648 \\
\hline D4A5K8 & Protein RGD1559600 GN=RGD1559600 & 0.647 \\
\hline Q6XQG8 & Carbohydrate sulfotransferase 7 GN=Chst7 & 0.646 \\
\hline Q642A3 & Transmembrane protein ENSP00000340100 homolog & 0.645 \\
\hline D4AA06 & Nuclear receptor binding SET domain protein 1 (Predicted), isoform CRA_a GN=Nsd1 & 0.643 \\
\hline Q5XIL2 & Phosphatidylinositol 4-kinase type 2-beta GN=Pi4k2b & 0.64 \\
\hline Q66HA1 & Mitogen-activated protein kinase kinase kinase 11 GN=Map3k11 & 0.64 \\
\hline D3Z8M1 & Olfactory receptor $\mathrm{GN}=\mathrm{Olr} 1452$ & 0.638 \\
\hline D4ABA9 & Protein LOC100910104 GN=LOC100910104 & 0.636 \\
\hline Q5BJN2 & Death-associated kinase 2 GN=Dapk2 & 0.635 \\
\hline D3ZX13 & Protein Kif20b GN=Kif20b & 0.631 \\
\hline D3ZIF5 & $\begin{array}{l}\text { Adaptor-related protein complex 3, beta } 1 \text { subunit (Predicted), isoform CRA_b } \\
\text { GN=Ap3b1 }\end{array}$ & 0.63 \\
\hline D4AA41 & $\begin{array}{l}\text { Endothelial differentiation, lysophosphatidic acid G-protein-coupled receptor } 4 \\
\text { (Predicted), isoform CRA_b GN=Pbx4 }\end{array}$ & 0.63 \\
\hline D4A817 & Histone H2B GN=Hist1h2bh & 0.629 \\
\hline Q56A26 & Oligodendrocytic myelin paranodal and inner loop protein $\mathrm{GN}=$ Opalin & 0.629 \\
\hline Q3B7D7 & Atg $4 \mathrm{~d}$ protein $\mathrm{GN}=\mathrm{Atg} 4 \mathrm{~d}$ & 0.626 \\
\hline F1LXT8 & Protein Dnah6 (Fragment) GN=Dnah6 & 0.625 \\
\hline D3ZG83 & Protein Map3k10 GN=Map3k10 & 0.624 \\
\hline D4A6E4 & Protein Epc1 (Fragment) GN=Epc1 & 0.622 \\
\hline D3ZDN7 & Protein Fgd6 GN=Fgd6 & 0.62 \\
\hline D3ZS95 & Protein Dpy1914 (Fragment) GN=Dpy1914 & 0.619 \\
\hline D4A823 & Protein Rdm1 GN=Rdm1 & 0.617 \\
\hline Q4FZY2 & Protein Sirt6 GN=Sirt6 & 0.614 \\
\hline B4F7E5 & ATPase, $\mathrm{Ca}++$ transporting, cardiac muscle, fast twitch $1 \mathrm{GN}=\mathrm{Atp} 2 \mathrm{a} 1$ & 0.611 \\
\hline D4A949 & Protein RGD1560303 GN=RGD1560303 & 0.61 \\
\hline Q7TPJ9 & Ac2-141 GN=rCG_63673 & 0.61 \\
\hline D3ZQ18 & Protein Nccrp1 GN=Nccrp1 & 0.609 \\
\hline F1M392 & Protein Limch1 GN=Limch1 & 0.604 \\
\hline P11517 & Hemoglobin subunit beta-2 & 0.603 \\
\hline D4A6B3 & Protein Slamf6 GN=Slamf6 & 0.603 \\
\hline Q56A21 & LOC365476 protein (Fragment) GN=Wdr96 & 0.599 \\
\hline Q5M949 & Nipsnap homolog 3A (C. elegans) GN=Nipsnap3b & 0.597 \\
\hline F1LTG2 & Protein Alpk2 GN=Alpk2 & 0.594 \\
\hline M0R406 & Protein Ppp4r4 GN=Ppp4r4 & 0.593 \\
\hline Q66HD3 & Nuclear autoantigenic sperm protein GN=Nasp & 0.593 \\
\hline D4A518 & Protein Dennd4a GN=Dennd4a & 0.593 \\
\hline D4A9H8 & Protein Nim1 GN=Nim1 & 0.588 \\
\hline D3ZRK7 & Cell division cycle 6 homolog (S. cerevisiae) (Predicted), isoform CRA_aGN=Cdc6 & 0.587 \\
\hline
\end{tabular}




\begin{tabular}{|c|c|c|}
\hline Q9WUI9 & Nuclear receptor coactivator $2 \mathrm{GN}=\mathrm{Ncoa} 2$ & 0.584 \\
\hline P63182 & Cerebellin-1 GN=Cbln1 & 0.583 \\
\hline Q8QZZ9 & Cyclin-dependent kinase inhibitor $2 \mathrm{~A}$, isoform $2 \mathrm{GN}=\mathrm{Cdkn} 2 \mathrm{a}$ & 0.578 \\
\hline B0BN02 & Mtx1 protein $\mathrm{GN}=\mathrm{Mtx} 1$ & 0.577 \\
\hline F1M6D4 & Protein Flt3 (Fragment) GN=Flt3 & 0.577 \\
\hline D3Z9L0 & Protein Agk GN=Agk & 0.577 \\
\hline Q3T1I2 & Complement $\mathrm{C} 1 \mathrm{q}$ tumor necrosis factor-related protein $6 \mathrm{GN}=\mathrm{C} 1 \mathrm{qtnf} 6$ & 0.576 \\
\hline Q66H58 & von Willebrand factor A domain-containing protein $9 \mathrm{GN}=\mathrm{Vwa} 9$ & 0.565 \\
\hline D3ZSL5 & Protein Ptpn14 GN=Ptpn14 & 0.563 \\
\hline Q9QUH3 & Apolipoprotein A-V GN=Apoa5 & 0.558 \\
\hline D4A497 & Protein Pcdh11x GN=Pcdh11x & 0.556 \\
\hline D4A254 & Protein Phlpp2 GN=Phlpp2 & 0.551 \\
\hline P84889 & Vang-like protein $2 \mathrm{GN}=$ Vangl2 & 0.547 \\
\hline Q5M9H1 & Leucine-rich repeat-containing protein $41 \mathrm{GN}=\mathrm{Lrrc} 41$ & 0.546 \\
\hline D3ZQG0 & Protein Rnmtl1 GN=Rnmtl1 & 0.545 \\
\hline Q63418 & Protocadherin-3 GN=Pcdh3 & 0.54 \\
\hline D3ZFX9 & Protein RGD1560207 GN=RGD1560207 & 0.538 \\
\hline Q63683 & Dual specificity protein phosphatase GN=Dusp1 & 0.535 \\
\hline Q63111 & Carcinoembryonic antigen-related cell adhesion molecule $3 \mathrm{GN}=$ Ceacam 3 & 0.529 \\
\hline D4A182 & Naked cuticle 2 homolog (Drosophila) (Predicted) GN=Nkd2 & 0.529 \\
\hline F1M376 & Olfactory receptor (Fragment) $\mathrm{GN}=\mathrm{Olfr} 1102$ & 0.525 \\
\hline Q5FWT8 & REST corepressor 2 GN=Rcor 2 & 0.516 \\
\hline D4A3X0 & Protein Seh11 GN=Seh11 & 0.506 \\
\hline D4A4L5 & Protein Isca2 GN=Isca2 & 0.5 \\
\hline G3V8U6 & DNA polymerase alpha subunit $\mathrm{B}$ GN=Pola2 & 0.481 \\
\hline B5DEY4 & Mitochondrial ribosomal protein $\mathrm{L} 30 \mathrm{GN}=\mathrm{Mrpl} 30$ & 0.478 \\
\hline D4A4T7 & Protein Dsg1 GN=Dsg1 & 0.45 \\
\hline D3ZEF4 & Cullin-7 GN=Cul7 & 0.412 \\
\hline
\end{tabular}

